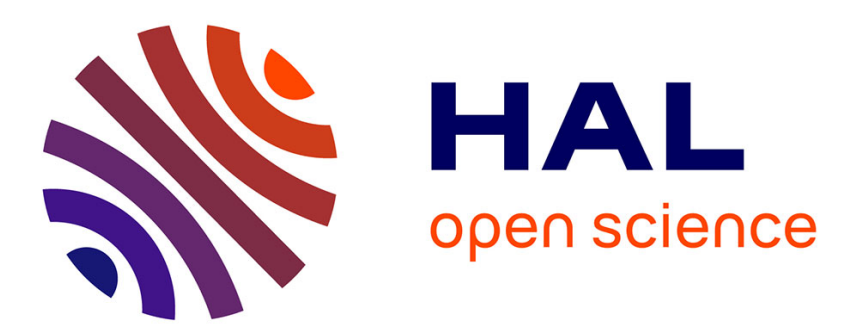

\title{
Vertex decompositions of sparse graphs into an edgeless subgraph and a subgraph of maximum degree at most $k$
}

O.V. Borodin, A.O. Ivanova, Mickael Montassier, Pascal Ochem, André Raspaud

\section{- To cite this version:}

O.V. Borodin, A.O. Ivanova, Mickael Montassier, Pascal Ochem, André Raspaud. Vertex decompositions of sparse graphs into an edgeless subgraph and a subgraph of maximum degree at most $\mathrm{k}$. Journal of Graph Theory, 2010, 65 (2), pp.83-93. 10.1002/jgt.20467 . hal-00377372

\section{HAL Id: hal-00377372 \\ https://hal.science/hal-00377372}

Submitted on 21 Apr 2009

HAL is a multi-disciplinary open access archive for the deposit and dissemination of scientific research documents, whether they are published or not. The documents may come from teaching and research institutions in France or abroad, or from public or private research centers.
L'archive ouverte pluridisciplinaire HAL, est destinée au dépôt et à la diffusion de documents scientifiques de niveau recherche, publiés ou non, émanant des établissements d'enseignement et de recherche français ou étrangers, des laboratoires publics ou privés. 


\title{
Vertex decompositions of sparse graphs into an edgeless subgraph and a subgraph of maximum degree at most $k$
}

\author{
O. V. Borodin, \\ Institute of Mathematics and Novosibirsk State University, Novosibirsk, 630090, Russia
}

A. O. Ivanova

Institute of Mathematics at Yakutsk State University, Yakutsk, 677891, Russia. *

M. Montassier, and A. Raspaud

LaBRI, Université Bordeaux I, 33405 Talence Cedex, France

March 17, 2009

\begin{abstract}
A graph $G$ is $(k, 0)$-colorable if its vertices can be partitioned into subsets $V_{1}$ and $V_{2}$ such that in $G\left[V_{1}\right]$ every vertex has degree at most $k$, while $G\left[V_{2}\right]$ is edgeless. For every integer $k \geq 1$, we prove that every graph with the maximum average degree smaller than $\frac{3 k+4}{k+2}$ is $(k, 0)$-colorable. In particular, it follows that every planar graph with girth at least 7 is $(8,0)$-colorable. On the other hand, we construct planar graphs with girth 6 that are not $(k, 0)$-colorable for arbitrarily large $k$.
\end{abstract}

\section{Introduction}

A graph $G$ is called improperly $\left(d_{1}, \ldots, d_{k}\right)$-colorable, or just $\left(d_{1}, \ldots, d_{k}\right)$-colorable, if the vertex set of $G$ can be partitioned into subsets $V_{1}, \ldots, V_{k}$ such that the graph $G\left[V_{i}\right]$ induced by the vertices of $V_{i}$ has maximum degree at most $d_{i}$ for all $1 \leq i \leq k$. This notion generalizes those of proper $k$-coloring (when $d_{1}=\ldots=d_{k}=0$ ) and $d$-improper $k$-coloring (when $d_{1}=\ldots=d_{k}=d \geq 1$ ).

Proper and $d$-improper colorings have been widely studied. As shown by Appel and Haken [1,2], every planar graph is 4-colorable, i.e. (0, 0, 0, 0)-colorable. Eaton and Hull [11] and independently Škrekovski [15] proved that every planar graph is 2-improperly 3-colorable (in fact, 2-improper choosable), i.e. $(2,2,2)$-colorable. This latter result was extended by Havet and Sereni [14] to not necessarily planar sparse graphs.

Theorem 1 [14] For every $k \geq 0$, every graph $G$ with $\operatorname{mad}(G)<\frac{4 k+4}{k+2}$ is $k$-improperly 2-colorable (in fact $k$-improperly 2-choosable), i.e. $(k, k)$-colorable.

Recall that $\operatorname{mad}(G)=\max \left\{\frac{2|E(H)|}{|V(H)|}, H \subseteq G\right\}$ is the maximum average degree of a graph $G$.

In this paper, we focus on $(k, 0)$-colorability of graph. So, a graph $G$ is $(k, 0)$-colorable if its vertices can be partitioned into subsets $V_{1}$ and $V_{2}$ such that in $G\left[V_{1}\right]$ every vertex has degree at most $k$, while $G\left[V_{2}\right]$ is edgeless.

Let $g(G)$ denote the girth of graph $G$ (the length of the shortest cycle in $G$ ). Glebov and Zambalaeva [12] proved that every planar graph $G$ is $(1,0)$-colorable if $g(G) \geq 16$. This was strengthened

\footnotetext{
* The first and second authors were supported by grants 06-01-00694 and 08-01-00673 of the Russian Foundation for Basic Research, the second author was also supported by the President of Russia grant for young scientists MK-2302.2008.1.
} 
by Borodin and Ivanova [6] by proving that every graph $G$ is $(1,0)$-colorable if $\operatorname{mad}(G)<\frac{7}{3}$, which implies that every planar graph $G$ is $(1,0)$-colorable if $g(G) \geq 14$.

The purpose of our paper is to extend the result in [6] as follows:

Theorem 2 Let $k \geq 1$ be a integer. Every graph with maximum average degree smaller than $\frac{3 k+4}{k+2}$ is $(k, 0)$-colorable.

On the other hand, we construct non- $(k, 0)$-colorable graphs whose maximum average degree exceeds $\frac{3 k+4}{k+2}$ not much (in fact, by less than $\frac{1}{k+3}$, see Section 3 ).

Since each planar graph $G$ satisfies

$$
\operatorname{mad}(G)<\frac{2 g(G)}{g(G)-2},
$$

from Theorem 2 we have:

Corollary 1 Every planar graph $G$ is:

1. (1,0)-colorable if $g(G) \geq 14$,

2. (2,0)-colorable if $g(G) \geq 10$,

3. (3,0)-colorable if $g(G) \geq 9$,

4. (4,0)-colorable if $g(G) \geq 8$, and

5. $(8,0)$-colorable if $g(G) \geq 7$.

Note that Corollary 1.5 is best possible in the sense that for any $k \geq 1$ there is a planar graph with girth 6 which fails to be $(k, 0)$-colorable:

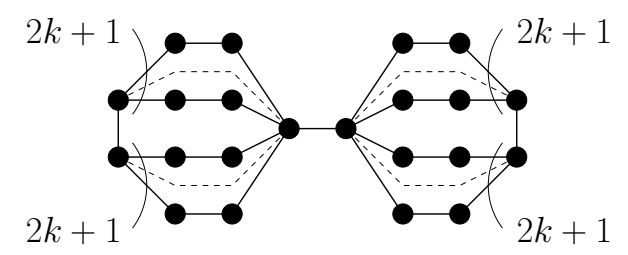

Figure 1: A non $(k, 0)$-colorable graph $G$ with girth 6.

The key concepts in the proof of our main result, Theorem 2, are those of soft component and feeding area. These further develop those of soft cycle and feeding path introduced by Borodin, Ivanova, Kostochka in [7] and used in [7, 5] to improve results in [10, 8] about homomorphisms of sparse graphs to the circulant $C(5 ; 1,2)$ and cycle $C_{5}$. A distinctive feature of the discharging in $[7,5]$ is that charge can be transferred along "feeding paths" to an unlimited distance. Note that similar ideas of "global discharging" were also used by Havet and Sereni in [14]. In fact, the proof of Theorem 2 below further develops the argument in [6].

An induced cycle $C=v_{1} v_{2} \ldots v_{2 k}$ in a graph $G$ is called 2-alternating if $d\left(v_{1}\right)=d\left(v_{3}\right)=\ldots=$ $d\left(v_{2 k-1}\right)=2$. This is perhaps the first and simplest example of a "global" reducible configuration in some graph-theoretic problems. The feeding area in this case is a tree consisting of edges incident with vertices of degree 2. This notion, introduced by Borodin in [3], as well as its subsequent variations, turned out to be useful in some coloring and edge-decomposition problems on sparse and quasiplanar graphs (see, for example, [9, 4, 13, 16, 17]).

Section 2 is dedicated to the proof of Theorem 2. Section 3 contains some final remarks. 


\section{Proof of Theorem 2}

Let $G$ be a minimal counterexample to Theorem 1. Due to [6], we can assume that $k \geq 2$. Furthermore, the proofs below for $k=2$ and $k \geq 3$ will differ a little (namely, in the treatment of $(1,1,1,0)$-vertex, which will be defined later). [Words concerning only case $k=2$ appear three or four times, in brackets.]

Clearly, $G$ is connected and its minimum degree $\delta(G)$ is at least 2. By definition, we have

$$
\sum_{v \in V}\left(d(v)-\frac{3 k+4}{k+2}\right)<0,
$$

where $d(v)$ is the degree of a vertex $v$. This can be rewritten as

$$
\sum_{v \in V}\left(\left(2+\frac{4}{k}\right) d(v)-\left(6+\frac{8}{k}\right)\right)<0 .
$$

Let the charge $\mu(v)$ of each vertex $v$ of $G$ be $\left(2+\frac{4}{k}\right) d(v)-\left(6+\frac{8}{k}\right)$. Since $\delta(G) \geq 2$, it follows that in $G$ only 2-vertices have negative charge (equal to -2 ). We shall describe a number of structural properties of $G$ (Section 2.1) which make it possible to vary the charges so that the new charge $\mu^{*}$ of every vertex becomes nonnegative (Section 2.2). Since the sum of charges does not change, we shall get a contradiction with (1), which will complete the proof of Theorem 2 .

In what follows, by a $k$-path we mean a path consisting of precisely $k$ vertices of degree 2 , while by a $\left(k_{1}, k_{2}, \ldots\right)$-vertex we mean a vertex that is incident with $k_{1^{-}}, k_{2}-, \ldots$ paths. A vertex of degree $k$ (resp. at least $k$, at most $k$ ) is called a $k$-vertex (resp. $\geq k$-vertex, $\leq k$-vertex). We will color the vertices of the independent set by the color 0 , and the vertices of the subgraph of maximum degree at most $k$ by the color $k$.

A vertex of degree at least $k+2$ is called senior. Note that if a vertex $v$ is senior, then

$$
\mu(v)=\left(2+\frac{4}{k}\right) d(v)-\left(6+\frac{8}{k}\right) \geq 2 d(v)+\frac{4}{k}(k+2)-6-\frac{8}{k}=2 d(v)-2 .
$$

\subsection{Structural properties}

Lemma 1 Every $\leq(k+1)$-vertex is adjacent to at least one senior vertex.

Proof. We delete such a vertex $v$ and extend a coloring $c$ of the graph obtained to $G$ as follows. If $v$ has all its neighbors colored with $k$, then we are done by putting $c(v)=0$. Now, if $v$ has at most $k$ neighbors colored with $k$, then we put $c(v)=k$. We are in trouble only if $v$ is adjacent to a $(k+1)$-vertex $x$ which is colored with $k$ together with all its neighbors. But then we can recolor $x$ with 0 . The same argument is then applicable to every other neighbor of $v$.

It follows that:

Corollary 2 G has no $\geq 3$-paths.

A triangle is special if has at least two 2-vertices. Since $G \neq C_{3}$, a special triangle actually has just two vertices of degree 2 .

Corollary 3 There is no $\geq 3$-vertex of degree at most $k+1$ incident with a 2-path, or a special triangle, or adjacent to a $(1,1,0)$-vertex. Moreover if $k \geq 3$, then there is no $\geq 3$-vertex of degree at most $k+1$ adjacent to $(1,1,1,0)$-vertex.

A vertex is bad if it is either a 2-vertex belonging to a 2-path or a $(1,1,0)$-vertex. Otherwise, a vertex is good. 
Lemma 2 Every $\geq 3$-vertex of $G$ has at least one good neighbor; furthermore, if $k \geq 3$ then it has a good neighbor that is not a $(1,1,1,0)$-vertex.

Proof. By contradiction, assume that a vertex $v$ of $G$ is not adjacent to good vertices. We delete the vertex $v$ and all its neighbors. To extend a coloring $c$ of the graph obtained to $G$, we color the vertex $v$ with 0 and color all neighbors of $v$ by $k$.

Corollary 4 G has no $(2,2, \ldots, 2)$-vertices.

By a soft vertex we mean a senior vertex whose each neighbor either has degree 2 , or is a $(1,1,0)$ vertex, or [unless $k=2$ ] a $(1,1,1,0)$-vertex. of:

By a feeding area, denoted by $F A$, we mean a maximal (by inclusion) subgraph in $G$ consisting

(i) soft vertices mutually accessible from each other along 1-paths, of

(ii) the following three types of vertices adjacent to soft vertices of $F A$ : 2-vertices, $(1,1,0)$ - and [unless $k=2$ ] $(1,1,1,0)$-vertices. See Figure 2.

(iii) 2-vertices belonging to those 1-paths whose both end-vertices belong to $F A$.

Note that according to this definition, every edge $x y$ joining $x \in F A$ with $y \notin F A$ has the following properties: (a) if $d(x)=2$, then $y$ is not soft ; (b) if $x$ is a $(1,1,0)$ - or [unless $k=2$ ] $(1,1,1,0)$-vertex, then $d(y)=2$.
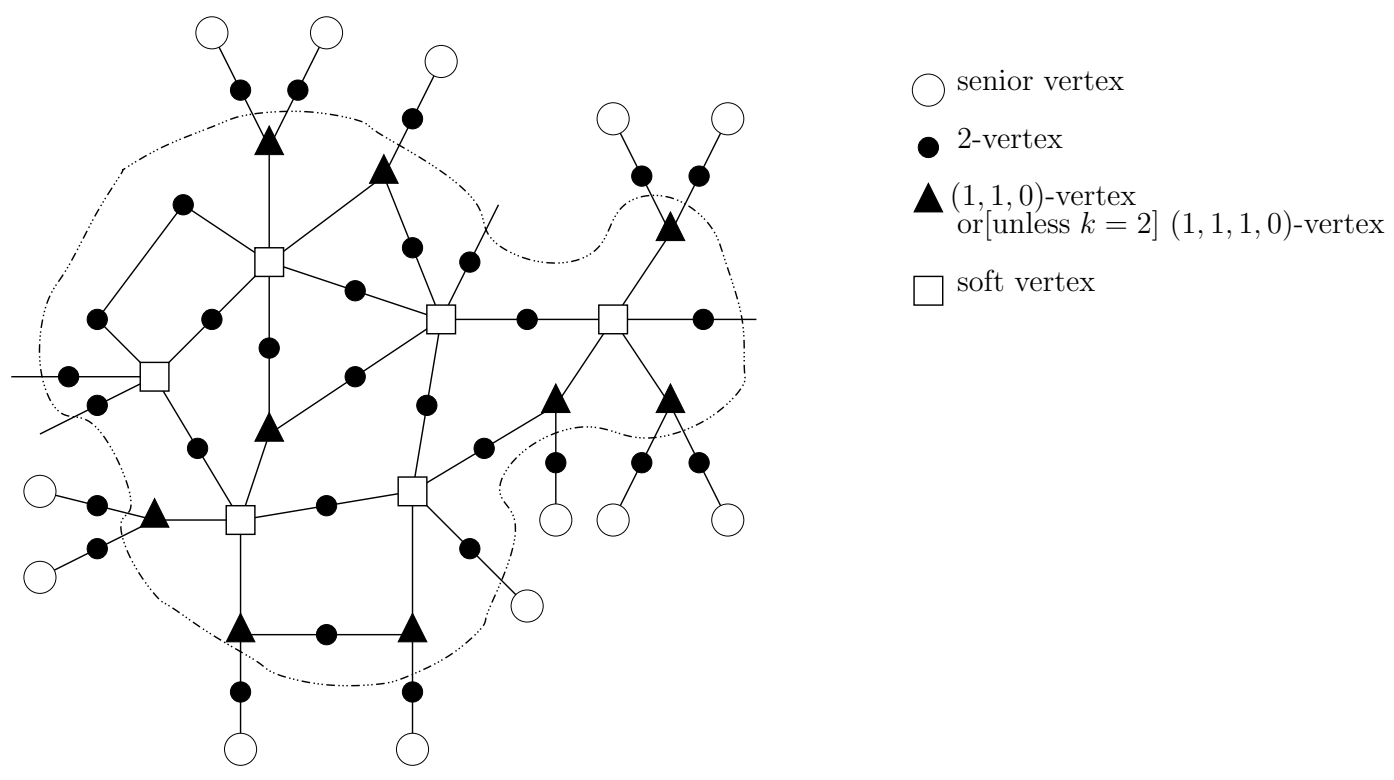

Figure 2: Example of a feeding area.

By a soft component we mean a feeding area $F A$ such that every edge from $F A$ to $G \backslash F A$ leads to a non-senior vertex.

Lemma 3 G has no soft components. 
Proof. Let $F A$ be a soft component. (It is not excluded that $F A=G$.) We first take a coloring $c$ of $G \backslash F A$. Now for each edge $x y$ such that $x \in F A$ and $y \notin F A$, we color $x$ with $k$. Now if $y$ is a $(k+1)$-vertex colored $k$ together with all its neighbors, we recolor $y$ with 0 . Then we color every soft vertex of $F A$ with 0 . Finally, we color all yet uncolored vertices of $F A$ (namely, 2-, $(1,1,0)$-, and [unless $k=2$ ] $(1,1,1,0)$-vertices adjacent only to vertices of $F A$ ) with $k$ to get a desired coloring of $G$.

Corollary 5 For each feeding area $F A$ of $G$ there exists a 1-path xyz such that $x \in F A$ is a soft vertex, while $y \in F A, z \notin F A$, where $z$ is a senior vertex incident with at least one 0-path.

A feeding area is weak, and denoted $W F A$, if it has only one 1-path $x y z$ such that $x \in F A$ is a soft vertex, while $z \notin F A$, where $z$ is a senior vertex incident with at least one 0 -path.

By a special vertex we mean a senior vertex $z$ such that:

(i) $z$ is incident with precisely one 0 -path going to a vertex which is neither $(1,1,0)$-vertex nor [unless $k=2$ ] $(1,1,1,0)$-vertex, and

(ii) every 1-path from $z$ leads to a $W F A$.

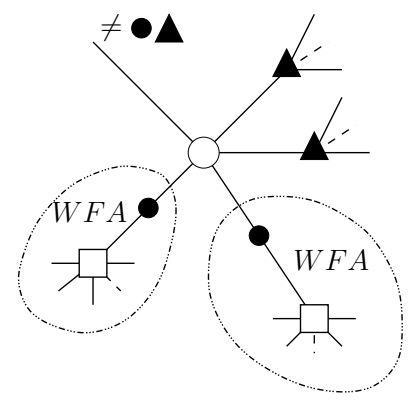

Figure 3: Example of a special vertex.

The notion of a special soft component, denoted $S S C$, is very close to that of a soft component. The only difference is that $S S C$ includes just one vertex, called its special vertex, that $S C$ fails to include. Namely, a special soft component $S S C$ consists of a special vertex $z$, all 2-, $(1,1,0)$-, and [unless $k=2$ ] $(1,1,1,0)$-vertices adjacent to $z$, and the vertices of all those $W F A$ 's joined to $z$ along their unique outgoing 1-paths. Informally speaking, a $S S C$ is a collection of $W F A$ 's joined by a special vertex.

Note that a smallest $S S C$ consists of its special vertex $z$ and $k+1$ adjacent 2 -vertices, all belonging to 2-paths incident with $z$. Furthermore, $z$ is incident with precisely one 0 -path $z x$, and $x$ is neither $(1,1,0)$-vertex nor [unless $k=2$ ] $(1,1,1,0)$-vertex.

Lemma 4 Each $(1,0,0)$-vertex $w$ is adjacent to a(t least one) senior non-special vertex.

Proof. Suppose a $(1,0,0)$-vertex $w$ is adjacent to $\geq 3$-vertices $z_{1}, z_{2}$ such that each $z_{i}$ is either a special vertex $z_{i} \in S S C_{i}$ or has $d\left(z_{i}\right) \leq k+1$, where $1 \leq i \leq 2$. Delete $w$; if $z_{i} \in S S C_{i}$ then also delete $S S C_{i}$ (together with $z_{i}$ ). We first take a coloring $c$ of the graph obtained and recolor the 2-vertex adjacent to $w$ with a color different from the color of its undeleted neighbor. Now if $z_{i} \in S S C_{i}$ then $S S C_{i}$ can be colored as in the proof of Lemma 5; in particular, each such $z_{i}$ is colored with 0 . Next, we color $w$ with $k$. Now, the only obstacle is that there is a $z_{i}$ such that $d\left(z_{i}\right)=k+1\left(z_{i}\right.$ is a non-senior vertex $)$ and $z_{i}$ is colored $k$ together with all its neighbors. However, then nothing prevents us from recoloring such a $z_{i}$ with 0 . 


\subsection{Discharging procedure}

Our rules of discharging are:

R1. Every 2-vertex that belongs to a 1-path gets charge 1 from its ends, while each 2 -vertex that belongs to a 2-path gets charge 2 from the neighbor vertex of degree greater than 2 .

R2. Each $(1,1,0)$-vertex gets charge 2 from its senior neighbor.

R3. If $k \geq 3$, then each $(1,1,1,0)$-vertex gets charge 1 from its senior neighbor.

R4. Each (1, 0,0)-vertex gets charge 1 from its senior non-special neighbor.

R5. Every weak FA gets charge 1 along its only 1-path that leads to $G \backslash F A$.

Lemma 5 The total charge $\mu^{*}(F A)$ of all soft vertices in each feeding area FA after applying rules R1-4 is nonnegative if FA is not weak and is at least -1 otherwise.

PROOF. We perform a series of transformations, each of which makes a feeding area $F A$ into another, "more standard", feeding area $F A^{\prime}$ of the same type (weak or otherwise) such that $\mu\left(F A^{\prime}\right) \leq \mu(F A)$. Eventually, any original feeding area $F A$ will be transformed into a(n ultimate) feeding area $F A_{0}$ which consists of a $(k+2)$-vertex $v$ whose all neighbors have degree 2 . Furthermore, if $F A_{0}$ is weak then precisely one of the neighbors of $v$ will belong to a 1-paths, which means that $\mu\left(F A_{0}\right) \geq-1$ in this case; if $F A_{0}$ is not weak then the number of 1-paths incident with $v$ will be at least 2 , so that $\mu\left(F A_{0}\right) \geq 0$. We reduce $F A$ to $F A_{0}$ as follows:

Step 1. If $F A$ has a cycle of 1-paths, then we replace one of its 1-paths by a 2-path and get a feeding area $F A^{\prime}$ such that $\mu\left(F A^{\prime}\right)=\mu(F A)-2$ due to $\mathrm{R} 1$. We repeat this procedure until all the cycles of 1-paths disappear.

Step 2. If $F A$ has a 2-path $P$ joining vertices $u, w$ of $F A$, then we replace $P$ by two 2-paths one of which is incident only with $u$, while the other only with $w$, so that the other ends of these new 2-paths "are loose" (do not belong to $F A^{\prime}$ ). As a result we have $\mu\left(F A^{\prime}\right)=\mu(F A)$.

Step 3. If $F A$ has a $(1,1,0)$-vertex $v$ joined to vertices $u$ and $w$ by 1 -paths, then we delete the 2neighbors of $v$. Now add a loose 1-path to $v$ and add a loose 2-path to each vertex in $\{u, w\} \cap$ $F A$. As a result we have $\mu\left(F A^{\prime}\right)=\mu(F A)-|\{u, w\} \cap F A| \leq \mu(F A)$ due to R1 and R2.

Step 4. If $F A$ has a $(1,1,1,0)$-vertex then arguing as in Case 3 , we have $\mu\left(F A^{\prime}\right)<\mu(F A)$ by R1 and R3.

From now on, our $F A$ is a tree consisting of senior (soft) vertices and 2-vertices with the property that each path between two senior vertices is a 1-path.

Step 5. Let $P_{i}=x_{i} y_{i} z_{i}, 1 \leq i \leq t$ be all 1-paths such that $x_{i} \in F A$ while $\left\{z_{i}\right\} \cap F A=\emptyset$. (Recall that $t \geq 1$ and $F A$ is weak if and only if $t=1$.) If $t \geq 3$ then we replace each $P_{i}$ with $i \geq 3$ by a 2-path $x_{i} y_{i} y_{i}^{\prime} z_{i}$. This results in $\mu\left(F A^{\prime}\right) \leq \mu(F A)$.

Note that in none of Steps 1-5 we changed the status of $F A$ to be weak or non-weak. The same is true for remaining two subcases.

Step 6. Suppose $F A$ has at least two senior vertices. Let $v$ be a pendent senior vertex in $F A$, i.e. joined by 1-path with precisely one senior vertex $w \in F A$. Suppose $v$ is incident with $p$ outgoing 1-paths. Since $F A$ has at least two pendant vertices and at most two outgoing 1-paths, we can assume that $p \leq 1$. If $p=0$ then we replace $v$ and its neighbors by a 2-path incident with $w$, which implies $\mu\left(F A^{\prime}\right)=\mu(F A)$. If $p=1$, we replace $v$ and and its neighbors by a loose 1-path going out of $w$, which again implies $\mu\left(F A^{\prime}\right)=\mu(F A)$ by $\mathrm{R} 1$. 
Step 7. If $v$ is the only senior vertex in FA and $d(v)>k+2$, then we make $v$ into a $(k+2)$-vertex by deleting incident 2-paths. This results in $\mu\left(F A^{\prime}\right)=\mu(F A)-2(d(v)-k-2)=2 k+2<$ $\mu(F A)$.

Finally, we obtain $F A_{0}$ with $\mu\left(F A_{0}\right)=-1$ is $F A_{0}$ is weak and with $\mu\left(F A_{0}\right) \geq 0$ otherwise.

We now check that after applying R1-R5, the new charge $\mu^{*}$ of each non-soft vertex $v$ and of each feeding area is nonnegative.

Indeed, if $d(v)=2$ then $\mu^{*}(v)=-2+2=0$ by R1 due to Corollary 2 .

Suppose $d(v)=3$. By Lemma $1, v$ is not a $(\geq 1, \geq 1, \geq 1)$-vertex and $v$ is not incident with a 2-path. If $v$ is a $(1,1,0)$-vertex then $\mu^{*}(v)=\frac{4}{k}-2 \times 1+2>0$ by $\mathrm{R} 1$ and $\mathrm{R} 2$ due to Lemma 1 . If $v$ is a $(1,0,0)$-vertex then $\mu^{*}(v)=\frac{4}{k}-1+1>0$ by R1 and R4 due to Lemma 4. Finally, if $v$ is a $(0,0,0)$-vertex then $\mu^{*}(v)=\frac{4}{k}>0$.

Next suppose $k \geq 3$ and $d(v)=4$. Now $\mu(v)=2+\frac{8}{k}>2$. By Lemma 1 and Corollary $3, v$ is adjacent to a senior vertex and is not incident with 2-paths. This implies that $\mu^{*}(v)=2+\frac{8}{k}-2 \times 1>$ 0 , unless $v$ is a $(1,1,1,0)$-vertex, in which case $\mu^{*}(v)=2+\frac{8}{k}-3 \times 1+1>0$ by R1 and R3 combined with Lemma 1.

Now suppose $k \geq 4$ and $5 \leq d(v) \leq k+1$. By Lemma 1 and Corollary $3, v$ gives 1 to at most $d(v)-1$ vertices of degree 2 by R1 and does not participate in other rules of discharging, which implies $\mu^{*}(v)>2(d(v)-3)-(d(v)-1) \times 1=d(v)-5 \geq 0$.

Finally, let $v$ be senior, i.e., having $d(v) \geq k+2$. Recall that $\mu(v) \geq 2 d(v)-2$. It follows from the first part of Lemma 2 that $v$ cannot give 2 along each incident path according to R1 and R2, which implies that $\mu^{*}(v) \geq \mu(v)-2(d(v)-1)-1 \geq-1$.

Suppose $v$ is not soft. If $v$ is special then $v$ does not give charge 1 along its (only) 0-path by $\mathrm{R} 4$, which implies that $v$ only gives away at most 2 along its $\geq 1$-paths by $\mathrm{R} 1$ and R5. Thus, $\mu^{*}(v) \geq \mu(v)-2(d(v)-1) \geq 0$.

It remains to consider a senior vertex $v$ which is neither soft nor special. Such a $v$ has either at least two 0 -paths that do not lead to $(1,1,0)$-vertices (and thus take away at most 1 from $v$ by R3 and R4), in which case $\mu^{*}(v) \geq \mu(v)-2(d(v)-2)-2 \times 1 \geq 0$, or has just one 0 -path that leads to a vertex other than $(1,0,0)-,(1,1,0)$ - or [unless $k=2$ ] $(1,1,1,0)$-vertex (so that this 0 -paths takes away no charge from $v)$, in which case $\mu^{*}(v) \geq \mu(v)-2(d(v)-1) \geq 0$.

By Lemma 5, after applying rules R1-R5, the total charge $\mu^{*}(F A)$ of all soft vertices in each feeding area $F A$ of $G$, both weak and non-weak, is nonnegative. Since the feeding areas are disjoint, it follows that the total $\mu^{*}$-charge of all soft vertices in $G$ is nonnegative.

This contradiction with (1) completes the proof of Theorem 2.

\section{Conclusion}

We proved that, for $k \geq 1$, every graph with maximum average degree smaller than $\frac{3 k+4}{k+2}$ is $(k, 0)$-colorable. We present now a non- $(k, 0)$-colorable graphs with small maximum average degree. Let $p, k \geq 1$ be two integers. Let $G_{p, k}$ be the graph obtained from an odd cycle $C_{2 p+1}=$ $x_{1} x_{2} \ldots x_{2 p+1} x_{1}$ by adding $k$ triangles on each vertex $x_{i}$ with odd $i$, as depicted in Figure 4 . 


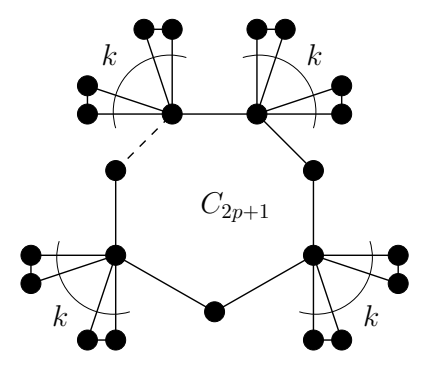

Figure 4: A non $(k, 0)$-colorable graph $G$ with small maximum average degree.

One can observe that $G_{p, k}$ is not $(k, 0)$-colorable. Indeed, suppose the contrary; then there should exist two consecutive vertices $v_{i}$ and $v_{i+1}$ in $C_{2 p+1}$ both belonging to $G\left[V_{1}\right]$ due to parity. W.1.o.g., let $i$ be odd; this implies that $v_{i}$ has at least one neighbor in $G\left[V_{1}\right]$ in each of $k$ incident triangles, so that $\Delta\left(G\left[V_{1}\right]\right) \geq k+1$, a contradiction.

Furthermore, by consecutively adding pendant triangles to $C_{2 p+1}$, we see that the average degree of $G_{p, k}$ is less than 3. The opposite process of removing vertices from $G_{p, k}$ then shows that $\operatorname{mad}\left(G_{p, k}\right) \geq \operatorname{mad}(H)$ for each $H \subseteq G_{p, k}$. Thus,

$$
\begin{gathered}
\operatorname{mad}\left(G_{p, k}\right)=\frac{2\left|E\left(G_{p, k}\right)\right|}{\left|V\left(G_{p, k}\right)\right|}=\frac{3 k+2-\frac{1}{p+1}}{k+1-\frac{1}{2(p+1)}}, \\
\lim _{p \rightarrow \infty} \operatorname{mad}\left(G_{p, k}\right)=\frac{3 k+2}{k+1}=3-\frac{1}{k+1}<\frac{3 k+4}{k+2}+\frac{1}{k+3} .
\end{gathered}
$$

Finally we propose the following conjecture:

Conjecture 1 Every graph with maximum average degree less than $\frac{3 k+2}{k+1}$ can be partitioned into an edgeless induced subgraph and an induced subgraph with maximum degree at most $k$.

\section{Acknowledgement}

The first author is thankful to the University of Bordeaux for inviting him as a visiting professor in the first half of 2009 and especially to Andre Raspaud for his cordial hospitality in Bordeaux.

\section{References}

[1] K. Appel and W. Haken, Every planar map is four colorable. Part I. Discharging. Illinois J. Math., 21 (1977) 429-490.

[2] K. Appel and W. Haken, Every planar map is four colorable. Part II. Reducibility Illinois J. Math., 21 (1977) 491-567.

[3] O. V. Borodin, On the total coloring of planar graphs, J. reine angew. Math., 394 (1989) 180-185.

[4] O.V. Borodin, A.O. Ivanova, and T.K. Neustroeva, Sufficient conditions for planar graphs with girth 6 to be 2-distance colourable, Siberian Electronic Math. Reports (http://semr.math.nsc.ru/), 3 (2006) 441-450 (in Russian).

[5] O.V. Borodin, S.G. Hartke, A.O. Ivanova, A.V. Kostochka, and D.B. West, $(5,2)$-Coloring of Sparse Graphs, Siberian Electronic Math. Reports, http://semr.math.nsc.ru, 5:417-426, 2008. 
[6] O.V. Borodin and A.O. Ivanova, Near-proper list vertex 2-colorings of sparse graphs, submitted.

[7] O.V. Borodin, A.O. Ivanova, and A.V. Kostochka, Oriented vertex 5-coloring of sparse graphs, Discrete analis and operations reseach, 13, no 1 (2006) 16-32 (in Russian).

[8] O.V. Borodin, S.J. Kim, A.V. Kostochka, and D.B. West, Homomorphisms of sparse graphs with large girth. J. of Combin. Theory B, 90 (2004) 147-159.

[9] O.V. Borodin, A.V.Kostochka, and D.R.Woodall, List edge and list total colourings of multigraphs, J. Combin. Theory (B) 71, 2 (1997) 184-204.

[10] O.V. Borodin, A.V. Kostochka, J. Nesetril, A. Raspaud, and E. Sopena, On the maximal average degree and the oriented chromatic number of a graph, Discrete Math., 206 (1999) 77-89.

[11] N. Eaton and T. Hull, Defective list colorings of planar graphs, Bull. Inst. Combin. Appl., 25 (1999) 79-87.

[12] A.N. Glebov, D.Zh. Zambalaeva, Path partitions of planar graphs, Siberian Electronic Math. Reports, http://semr.math.nsc.ru, 4 (2007) 450-459 (in Russian).

[13] W. He, X. Hou, K. W. Lih, J. Shao, W. Wang, and X. Zhu, Edge-partitions of planar graphs and their game coloring numbers, J. Graph Theory, 41 (2002) 307-317.

[14] F. Havet and J.-S. Sereni, Improper choosability of graphs and maximum average degree, Journal of Graph Theory, 52 (2006) 181-199.

[15] R. Škrekovski, List improper coloring of planar graphs, Comb. Prob. Comp., 8 (1999) 293-299.

[16] W. Wang, Edge-partitions of graphs of nonnegative characteristic and their game coloring number. Discrete Math., 2 (2006) 262-270.

[17] J.L. Wu, On the linear arboricity of planar graphs of nonnegative characteristic and their game coloring number, J. Graph Theory, 31 (1999) 129-134. 